\title{
Three-body QED test and fifth-force constraint from vibrations and rotations of $\mathrm{HD}^{+}$
}

\author{
M. Germann,,${ }^{1, *}$ S. Patra $\odot,{ }^{1, \dagger}$ J.-Ph. Karr $\odot,{ }^{2,3}$ L. Hilico $\odot,{ }^{2,3}$ V. I. Korobov $\odot,{ }^{4}$ E. J. Salumbides, ${ }^{1}$ K. S. E. Eikema $\odot,{ }^{1}$ \\ W. Ubachs $\odot,{ }^{1}$ and J. C. J. Koelemeij ${ }^{1, \ddagger}$ \\ ${ }^{1}$ LaserLaB, Department of Physics and Astronomy, Vrije Universiteit Amsterdam, De Boelelaan 1081, 1081 HV Amsterdam, The Netherlands \\ ${ }^{2}$ Laboratoire Kastler Brossel, Sorbonne Université, CNRS, ENS-PSL Research University, Collège de France, \\ 4 place Jussieu, 75005 Paris, France \\ ${ }^{3}$ Université d'Evry-Val d'Essonne, Université Paris-Saclay, Boulevard François Mitterrand, 91000 Evry, France \\ ${ }^{4}$ Bogoliubov Laboratory of Theoretical Physics, Joint Institute for Nuclear Research, Dubna 141980, Russia
}

(Received 19 February 2021; accepted 10 June 2021; published 28 June 2021)

\begin{abstract}
We present a parts-per-million test of quantum electrodynamics (QED) in the $\mathrm{HD}^{+}$molecular hydrogen ion, improving on previous tests based on vibrational and rotational transitions by factors of 76 and 1.4, respectively. The test is performed following a unified statistical approach that also produces improved constraints on physics beyond the standard model. We furthermore show how individual constraints derived from the various degrees of freedom in $\mathrm{HD}^{+}$and antiprotonic helium can be combined to enhance the sensitivity, thus ruling out "fifth forces” on the Ångstrom scale that are $10^{11}$ times weaker than the electromagnetic interaction.
\end{abstract}

DOI: 10.1103/PhysRevResearch.3.L022028

The standard model (SM) of particle physics is challenged by open questions concerning matter-antimatter asymmetry and the nature of dark matter and dark energy, which motivate searches for new physics (NP) beyond the standard model. Besides experiments at ever increasing interaction energies, precision measurements on particles, atoms, and molecules can provide low-energy SM tests through comparison with comparably accurate predictions obtained by relativistic quantum mechanics and quantum electrodynamics (QED). Deviations from QED predictions may indicate hitherto unknown physics beyond the SM, while agreement with observations allows us to constrain putative new physics [1].

As QED has withstood stringent tests at ever higher precision for single particles (sub-ppb [2-4]) and atoms (0.2 ppm $[5,6]$ ), one naturally asks whether it accurately describes more complex forms of matter, such as molecules, as well. Molecular systems, with their additional degrees of freedom, also open up new windows on possible NP [1,7]. In this Letter, we address these concepts in the revised landscape formed by recent extremely precise measurements of rotational [8] and vibrational [9] transition frequencies of the molecular hydrogen ion $\mathrm{HD}^{+}$, and of the masses of its constituent particles [10-14]. These results allow us to improve theoretical predictions, and test QED in rotating and vibrating systems with

\footnotetext{
*Present address: Department of Physics, Umeå University, 90187 Umeå, Sweden.

†resent address: Time and Frequency Division, National Institute of Standards and Technology, Boulder, Colorado 80305, USA.

¥j.c.j.koelemeij@vu.nl

Published by the American Physical Society under the terms of the Creative Commons Attribution 4.0 International license. Further distribution of this work must maintain attribution to the author(s) and the published article's title, journal citation, and DOI.
}

unprecedented sensitivity. We subsequently combine the new $\mathrm{HD}^{+}$results with data from another three-body system, $\overline{\mathrm{p}} \mathrm{He}$ $[15,16]$, to establish more stringent bounds on the strength and range of a possible internuclear "fifth force" [7].

Most previous tests of QED in $\mathrm{HD}^{+}$were carried out using either rovibrational transitions [17-20] or rotational transitions [21], observed via spectroscopy at the $\sim 1$-ppb level of trapped $\mathrm{HD}^{+}$ions cooled to $10 \mathrm{mK}$ by laser-cooled beryllium ions. Such tests were based on a qualitative assessment of the level of agreement between the experimental frequencies and frequency values predicted using nonrelativistic QED (NRQED) theory [22,23]. In some of these and other studies involving spectroscopy of light molecular species, the absence of large frequency differences between theory and experiment was interpreted as a constraint on NP such as Ångstromrange hadron-hadron interactions and compactified additional dimensions [7,8,19]. Fifth-force constraints exclude a region in the plane spanned by the range and coupling constant of the fifth force, and have so far been considered individually; i.e., no attempts have been made to combine data in order to increase the sensitivity and obtain an improved constraint.

A quantum leap in experimental accuracy was recently achieved in Doppler-free measurements in $\mathrm{HD}^{+}$of the $(v, L)$ : $(0,0) \rightarrow(0,1)$ fundamental rotational transition at $1.31 \mathrm{THz}$ [8], the $(v, L):(0,3) \rightarrow(9,3)$ vibrational overtone transition at $415 \mathrm{THz}$ [9], and the $(v, L):(0,0) \rightarrow(1,1)$ rotationalvibrational transition at $58.6 \mathrm{THz}$ [24] (here, $v$ and $L$ denote vibrational and rotational quantum numbers, respectively). Of these, only the rotational transition was used to test QED and constrain NP [8], where it was furthermore noted that the sensitivity was limited by the uncertainty of the CODATA-18 value of the proton-electron mass ratio, $m_{p} / m_{e}$, used in the theoretical calculation [25]. This eventually led Alighanbari et al. [8] as well as Patra et al. [9] and Kortunov et al. [24] to assume QED theory to be correct, and exploit theory to 
translate their precise measurement to an improved value of $m_{p} / m_{e}$.

Here, we present an improved test of molecular QED along with a more stringent fifth-force constraint that now also includes the $(v, L):(0,3) \rightarrow(9,3)$ transition which, at 3-ppt uncertainty, stands as the most accurate of all measurements performed on $\mathrm{HD}^{+}$so far (uncertainties of other measured spin-averaged frequencies are $13 \mathrm{ppt}[8]$ and $15 \mathrm{ppt}$ [24]). To this end we develop a unified statistical approach that ensures consistency between the QED test and the fifth-force constraint given prespecified confidence levels (CLs). Our test and constraint furthermore benefit from updated theoretical predictions reported in this work for both the rotational and vibrational transitions, taking advantage of strongly improved (and independent) values of $m_{p} / m_{e}$ and the deuteron-proton mass ratio $m_{d} / m_{p}$ [10-14] which reduce the uncertainty of the theoretical predictions by about $25 \%$. The theoretical predictions also include previously unevaluated terms of order $R_{\infty} \alpha^{5}$ and $R_{\infty} \alpha^{6}$ (with $R_{\infty}$ the Rydberg constant and $\alpha$ the fine-structure constant) [6,26-28], and a contribution from the deuteron polarizability $[6,26]$. These theory improvements are described in the Supplemental Material [29], where the resulting theoretical transition frequencies are listed together with the previous values. The new values have a relative uncertainty that approaches $1 \times 10^{-11}$, making them the most precise theoretical predictions for any molecular property published to date. We note that similar theory updates were reported very recently by Kortunov et al. for the $(v, L)$ : $(0,0) \rightarrow(1,1)$ vibrational transition [24]. However, in what follows we will focus primarily on the $(v, L):(0,3) \rightarrow(9,3)$ transition as a benchmark system for molecular vibrations, owing to its significantly higher experimental and theoretical accuracy (with the latter stemming from a lower sensitivity to the uncertainties of fundamental constants).

Figure 1 shows how the updated $(v, L):(0,3) \rightarrow(9,3)$ theoretical transition frequency, $v_{\text {theo }}$, is broken down into a nonrelativistic (NR) contribution as well as a number of relativistic and QED corrections (including nuclear recoil). Purely relativistic corrections [30,31] appear at even powers in $\alpha\left(R_{\infty} \alpha^{2}, R_{\infty} \alpha^{4}, R_{\infty} \alpha^{6}, \ldots\right.$, the latter contribution being negligibly small). QED corrections appear at order $R_{\infty} \alpha^{3}$ [32] and are considered up to order $R_{\infty} \alpha^{6}$ [22,30,33]. $v_{\text {theo }}$ also comprises a small correction to account for muonic and hadronic vacuum polarization. The NR term is calculated with negligible uncertainty by solving the three-body Schrödinger equation by a variational method [34,35]. Together with this part, we include a correction for the combined proton and deuteron finite nuclear size of $-830.6(5) \mathrm{kHz}$, computed using CODATA-18 values of the nuclear charge radii [25]. Besides the NR term, the leading-order relativistic $\left(R_{\infty} \alpha^{2}\right)$ and QED $\left(R_{\infty} \alpha^{3}\right)$ terms are also known with negligible uncertainty, and the theoretical uncertainty is caused almost entirely by the uncalculated $R_{\infty} \alpha^{6}$-order QED corrections (see inset of Fig. 1). The largest contribution to the total theoretical uncertainty is from the uncertainty of physical constants (mostly $\left.m_{p} / m_{e}\right)$ [9].

Experimental values of the $(v, L):(0,0) \rightarrow(0,1)$ rotational and $(v, L):(0,3) \rightarrow(9,3)$ vibrational transition frequencies have previously been obtained by Doppler-free terahertz and laser spectroscopy, respectively (see Refs. [8,9]

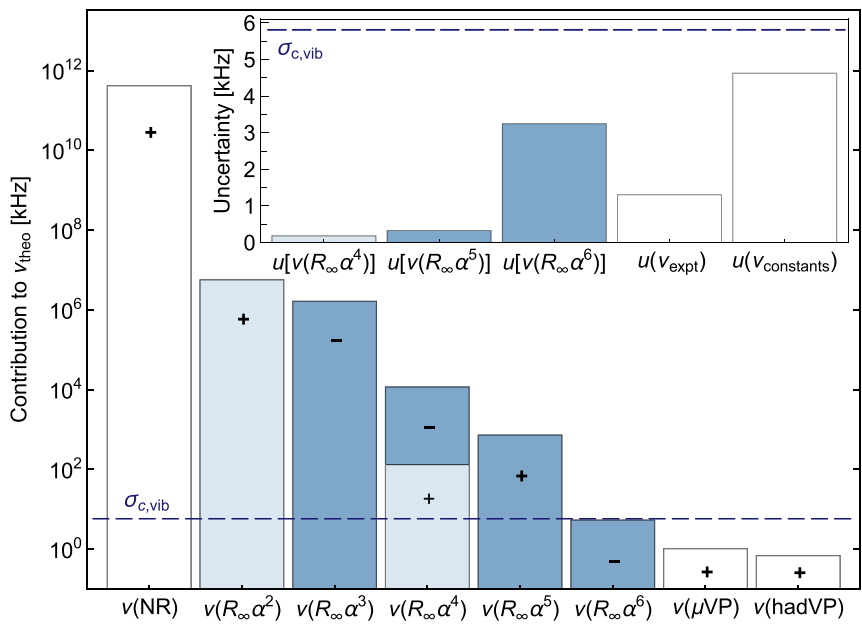

FIG. 1. Sizes and signs of contributions of order $R_{\infty} \alpha^{n}$ to the SM prediction of the $(v, L):(0,3) \rightarrow(9,3)$ transition frequency $v_{\text {theo }}$. Light blue, pure relativistic terms; dark blue, QED corrections. Contributions from muonic and hadronic vacuum polarization are also shown. The combined uncertainty (dashed horizontal line) is comparable to the $R_{\infty} \alpha^{6}$-order QED correction. Inset: Frequency uncertainties, $u$, of the contributions of orders $R_{\infty} \alpha^{4}$ through $R_{\infty} \alpha^{6}$ (the lower-order and NR terms are essentially exact). The experimental uncertainty, $u\left(v_{\text {expt }}\right)$, is small enough to test state-of-the-art three-body QED theory at its very limit. The quadratic sum of all uncertainties shown here, including the contribution from the physical constants, $u$ ( $\left.v_{\text {constants }}\right)$, equals $\sigma_{c \text {, vib }}$.

for details). These values correspond to spin-averaged frequencies; i.e., they were obtained after correcting measured transition frequencies of various hyperfine components for hyperfine shifts, which were found theoretically with sufficient precision. We here apply updated theoretical hyperfine shifts [36,37] which shift the experimental $(v, L):(0,3) \rightarrow(9,3)$ transition frequency upward by $1.3 \mathrm{kHz}$, and increase the uncertainty slightly from 1.2 to $1.3 \mathrm{kHz}$ [29]. For the $(v, L)$ : $(0,0) \rightarrow(0,1)$ transition frequency, the corresponding shift is negligibly small (less than $0.1 \sigma$ ), and we therefore retain the experimental frequency value reported by Alighanbari et al. [8]. These experimental values can be compared with the theoretical ones with a precision given by the combined uncertainty, $\sigma_{c}$, which comprises the uncertainties of the experiment, the physical constants used in the theory, and the uncertainties of the QED terms (Fig. 1). Given the relative uncertainties of the experimental rotational and vibrational overtone transition frequencies ( 13 and $3.1 \mathrm{ppt}$, respectively), molecular theory and experiment can now be compared at unprecedented relative combined uncertainties of $37 \mathrm{ppt}$ for the $(v, L):(0,0) \rightarrow(0,1)$ and $14 \mathrm{ppt}$ for the $(v, L):(0,3) \rightarrow$ $(9,3)$ transition.

To test the $\mathrm{HD}^{+}$theory we go beyond the qualitative character of previous QED tests [8,17-21] and set up a binary hypothesis test characterized by a significance level, $\alpha_{s}$, and statistical power, $1-\beta . \alpha_{s}$ represents the probability of a type-I error (i.e., inferring that an effect beyond the theory exists while in reality it does not exist), and $\beta$ the probability of a type-II error (i.e., judging the theory to be correct while a significant effect beyond the theory is present in reality). 
As explained in the Supplemental Material [29], we perform the test for $\alpha_{s}=0.0027$, thus checking for deviations between theory and experiment larger than $3 \sigma_{c}$ ). The found differences (experiment minus theory) are $-2.1 \sigma_{c, \text { rot }}$ and $-1.7 \sigma_{c, \text { vib }}$ for the rotational and vibrational transitions, respectively [29], leading us to conclude that at $99.7 \% \mathrm{CL}$, the molecular theory is valid for rotations and vibrations at the level $(3 \sigma)$ of 110 and $42 \mathrm{ppt}$, respectively. For the theory of molecular vibrations, this result is 76 times as stringent as the previous best test [19].

The hypothesis test carried out above can be also be interpreted as a test of the QED part of the calculation, which we will do with special attention to the $(v, L):(0,3) \rightarrow(9,3)$ transition which so far has not been considered in this context. The QED terms contribute in their entirety $-1.7 \mathrm{GHz}$ to the vibrational transition frequency, and the uncertainty in the theory stems almost entirely from the QED terms (Fig. 1). The agreement between theory and experiment found above can therefore be seen as a test of high-order molecular QED, based on a vibrational transition, at the level $(1 \sigma)$ of $(5.8 \mathrm{kHz}) /(1.7 \mathrm{GHz})=3.5 \mathrm{ppm}$. These findings represent a remarkable 76-fold improvement over the previous most precise QED test for molecular vibrations by Biesheuvel et al. [19], and the sensitivity may be further reduced to $\approx 0.3 \mathrm{ppm}$ if the precision of the theory and the constants are improved beyond the experimental uncertainty.

For the $(v, L):(0,0) \rightarrow(0,1)$ rotational transition in $\mathrm{HD}^{+}$ [8], our improved theoretical prediction improves the stringency of the QED test from 7.0 to $5.1 \mathrm{ppm}$ (at $1 \sigma$ ). It is worth noting that this test performed in a molecule exhibits qualitative differences with respect to those done, e.g., in hydrogenlike atoms, due to the existence of QED corrections specifically linked to the molecular nature of the system (see Supplemental Material [29] for details).

Our hypothesis test can also be used to derive bounds on possible frequency shifts caused by NP [29]. One advantage of this unified statistical approach is that the CL of such bounds is consistent with the CL specified for the QED test [29]. The location of the bound and the corresponding CL both depend on $\alpha_{s}$ and $\beta$, and for $\alpha_{s}=0.0027$ and $\beta=0.1$ we find a $90 \%-\mathrm{CL}$ constraint at $4.3 \sigma_{c}$ [29]. Our approach reveals that the CLs stated for some previous constraints were overestimated, with CLs being 50\% rather than the claimed 90\% [29]. In what follows we specifically consider hypothetical scalar hadron-hadron interactions at the 0.1-1 A length scale, which is typical for three-body systems like $\mathrm{HD}^{+}$and $\overline{\mathrm{p}} \mathrm{He}$. This range lies in between the scales investigated in highenergy and nuclear physics experiments, and those studied with atoms in weakly bound dimers [38] and macroscopic Cavendish-type setups. Following Salumbides et al. [7], we model such a hypothetical "fifth force" phenomenologically through a Yukawa-type potential,

$$
V_{\alpha_{5}, \lambda}(r)=\hbar c \alpha_{5} A_{1} A_{2} \frac{e^{-r / \lambda}}{r} .
$$

Here, $r$ is the separation of the two nuclei containing the interacting hadrons, $\alpha_{5}$ represents a dimensionless coupling constant, and $\hbar$ and $c$ are the reduced Planck constant and the speed of light, respectively. The range, $\lambda$, of the hypothetical interaction is related to the mass, $m_{5}$, of the corresponding hypothetical force carrier through $\lambda=\hbar /\left(m_{5} c\right)$. We treat the nucleon numbers $A_{1}$ and $A_{2}$ as charges of the hypothetical interaction.

The interaction in Eq. (1) leads to a first-order frequency shift of a transition $(v, L) \rightarrow\left(v^{\prime}, L^{\prime}\right)$,

$$
\begin{aligned}
\Delta v_{\alpha_{5}, \lambda}^{v v^{\prime} L L^{\prime}} & =\left(\Delta E_{\alpha_{5}, \lambda}^{v^{\prime} L^{\prime}}-\Delta E_{\alpha_{5}, \lambda}^{v L}\right) /(2 \pi \hbar) \\
& \equiv \alpha_{5} A_{1} A_{2} \mathcal{Y}_{\lambda}^{v v^{\prime} L L^{\prime}},
\end{aligned}
$$

where $\Delta E_{\alpha_{5}, \lambda}^{v L}=\left\langle v L\left|V_{\alpha_{5}, \lambda}\right| v L\right\rangle$, and $\mathcal{Y}_{\lambda}^{v v^{\prime} L L^{\prime}}$ is a normalized frequency shift that was computed on a dense grid of $\lambda$ values for relevant transitions in $\mathrm{HD}^{+}$and $\overline{\mathrm{p}} \mathrm{He}$ [7,29]. If theory and experiment are found to be in agreement, our hypothesis test constrains, for a given $\lambda$, the NP parameter $\left|\alpha_{5}\right|$ through the inequality

$$
\left|\alpha_{5}\right|<\left|\frac{4.3 \sigma_{c}}{A_{1} A_{2} \mathcal{Y}_{\lambda}{ }^{v v^{\prime} L L^{\prime}}}\right| .
$$

We apply Eq. (3) to the three recent measurements in $\mathrm{HD}^{+}$ $[8,9,24]$, as well as four transitions in $\bar{p}^{3} \mathrm{He}$ and $\bar{p}^{4} \mathrm{He}[15,16]$, which is justified as no deviations beyond $3 \sigma$ are observed for these transitions. The resulting 90\%-CL constraints are shown in Fig. 2.

The various transitions in Fig. 2 have diverse sensitivities with respect to $\lambda$. In the Supplemental Material [29] we show that in the (hypothetical) case of a detectable fifth-force frequency shift, the additional degrees of freedom offered by the various transitions and three-body systems studied here can be used to obtain separate estimates of the coupling constant as well as the range of a fifth force. It is also worth noting that the sensitivities $\mathcal{Y}_{\lambda}{ }^{v v^{\prime} L L^{\prime}}$ of vibrational transitions change sign at shorter range (corresponding to the peaks in the constraints shown in Fig. 2), which can lead to an increased differential fifth-force sensitivity among different transitions, and thereby enhance the resolution at which the range of a hypothetical fifth force may be determined [29].

Further, the above data can be combined into a single, more severe constraint. To this end, we fit $\alpha_{5}$ (for given $\lambda$ ) to multiple data simultaneously, through a least-squares optimization. At long range, the most constraining input data are the three recent $\mathrm{HD}^{+}$measurements. The corresponding constraints are partially correlated through the QED theory and the (correlated) physical constants used; all these correlations are taken into account [29]. To determine the combined constraint, we proceed in the same spirit as our treatment of single comparisons above. The least-squares fit produces an estimate $\hat{\alpha}_{5}$ with $1 \sigma$ uncertainty $u\left(\hat{\alpha}_{5}\right)$. We again carry out a hypothesis test, this time based on the value of $\hat{\alpha}_{5} / u\left(\hat{\alpha}_{5}\right)$. We find no deviations at the $3 \sigma$ level, and the corresponding $90 \%$ $\mathrm{CL}\left(4.3 \sigma_{c}\right)$ constraint is more stringent (by up to a factor of 3 ) than any of the individual constraints (Fig. 2). The impact of correlations is also visible in Fig. 2. The combined constraint clearly benefits from the multiple $\mathrm{HD}^{+}$and (uncorrelated) $\overline{\mathrm{p}} \mathrm{He}$ data near $\lambda=0.15 \AA$, but the strong correlation between the $\mathrm{HD}^{+}$rotational and vibrational data prevents a significant improvement near $\lambda=0.45 \AA$. We expect our approach to become useful for future NP searches as it is generally applicable and may incorporate any number of constraints, obtained from any system. 


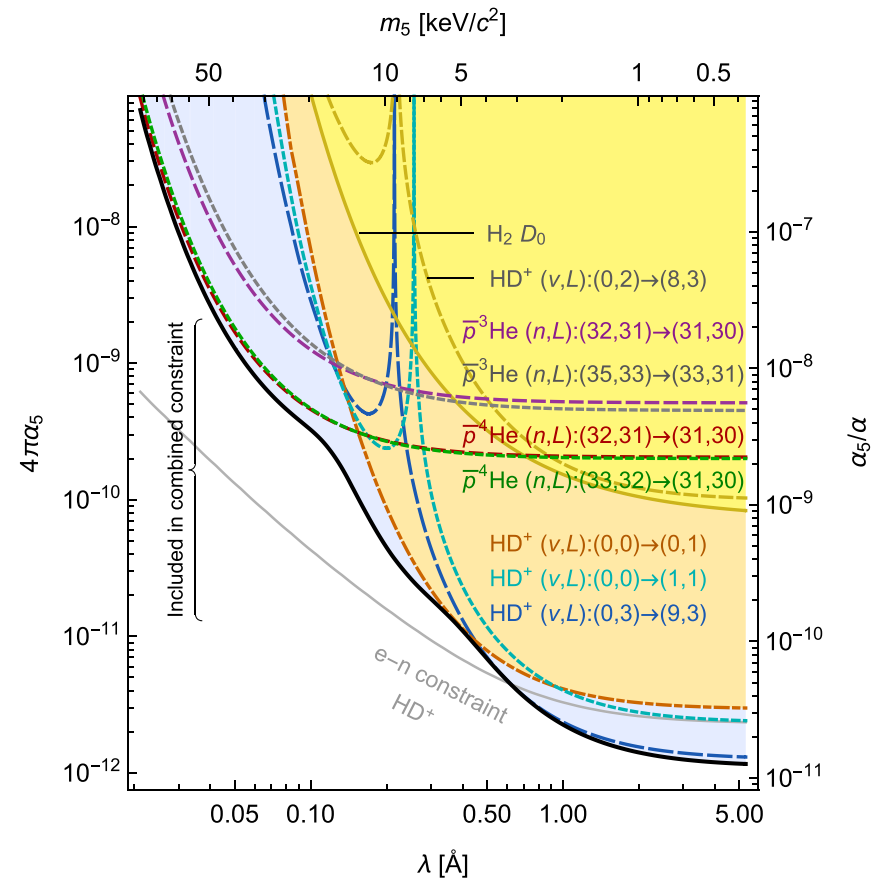

FIG. 2. 90\%-CL constraints on scalar hadron-hadron interactions inferred from a hypothesis test with significance level 0.0027 . Individual constraints are obtained from rotational and vibrational spectroscopy of $\mathrm{HD}^{+}$and from studies of $\overline{\mathrm{p}}^{3} \mathrm{He}$ and $\overline{\mathrm{p}}^{4} \mathrm{He}$ $[8,9,15,16,24]$. Black curve: Combined constraint obtained from a least-squares adjustment involving seven transitions. The upper yellow region indicates $\left(\lambda, \alpha_{5}\right)$ values excluded by the $\mathrm{H}_{2}$ dissociation limit (solid yellow curve [39]) and the previous best constraint from vibrational spectroscopy of $\mathrm{HD}^{+}$(dashed yellow curve [19]). Below, the orange area represents the parameter space excluded by the work of Alighanbari et al. [8]. Our combined constraint expands the excluded area further into the light blue region. The solid gray curve illustrates the constraint on a hypothetical electron-neutron coupling based on the three recent $\mathrm{HD}^{+}$measurements $[8,9,24]$.

At long range $(\lambda>5 \AA)$, we find $4 \pi \alpha_{5}<1 \times 10^{-12}$ at 90\% CL. It is interesting to compare this with results from other NP searches on the atomic scale. One example is the search for Yukawa-type electron-neutron $(e-n)$ couplings by analysis of data from isotope-shift spectroscopy and so-called King plots [40-43]. Such $e-n$ constraints cannot be directly compared with our nucleon-nucleon constraint; however, we have also computed $e-n$ Yukawa matrix elements similar to those in Eq. (2) by integrating $\hbar c \alpha_{5} \exp \left(-r_{d e} / \lambda\right) / r_{d e}$, with $r_{d e}$ the deuteron-electron distance, over the nonrelativistic threebody wave functions. We use these matrix elements to derive constraints from the three most recent $\mathrm{HD}^{+}$measurements, and inserting these constraints in a separate adjustment (assuming no electron-proton or neutron-proton coupling) leads to the $e-n$ constraint shown in Fig. 2. This constraint is nearly two orders of magnitude more stringent than the recent Kingplot results [42,43], and within one order of magnitude from the more stringent bound obtained from hydrogen-deuterium isotope shift spectroscopy [41]. However, it must be realized that our $e-n$ constraint depends linearly on $R_{\infty}$, whose value is determined from spectroscopy of hydrogenlike atoms [25] which might be affected by the very $e-n$ coupling we are trying to constrain. Note that this is not an issue for the Ångstrom-range hadron-hadron (or nucleon-nucleon) interactions considered in this work, since such interactions are absent in atomic systems. Future work in this direction should consider also the effect of NP on the physical constants used in the theory.

We may compare our nucleon-nucleon constraints with those inferred from neutron scattering $[29,44,45]$, which are still two orders of magnitude more stringent than ours. However, a crucial difference is that our models for $\mathrm{HD}^{+}$and $\overline{\mathrm{p}} \mathrm{He}$, as search grounds for NP, are built up from first principles and do not rely on model assumptions except those of relativistic quantum mechanics and QED. A null result obtained with these systems can therefore constrain a wider class of possible NP phenomena. For example, King-plot searches for NP in atoms are necessarily restricted to electron-neutron interactions subject to certain model assumptions regarding atomic and nuclear structure [41-43], whereas our systems can be used to constrain electron-neutron as well as electron-proton and proton-neutron interactions in a model-independent way. We thus conclude that our work not only rules out scalar hadron-hadron interactions at the Ångstrom scale that are $1 \times 10^{11}$ times weaker than the electromagnetic interaction (Fig. 2), but generally excludes NP that would modify the frequencies of molecular vibrations and rotations in $\mathrm{HD}^{+}$in the 11th decimal place, thereby providing evidence for the validity of the SM (and QED in particular) in molecules at the same level.

We acknowledge support from the Netherlands Organisation for Scientific Research (FOM Programs "Broken Mirrors \& Drifting Constants" and "The Mysterious Size of the Proton"; FOM 13PR3109, STW Vidi 12346), the European Research Council (AdG 670168 Ubachs, AdG 695677 Eikema), the COST Action CA17113 TIPICQA, and the Dutch-French bilateral Van Gogh program. V.I.K. acknowledges support from the Russian Foundation for Basic Research under Grant No. 19-02-00058-a.
[1] M. S. Safronova, D. Budker, D. DeMille, Derek F. Jackson Kimball, A. Derevianko, and C. W. Clark, Search for new physics with atoms and molecules, Rev. Mod. Phys. 90, 025008 (2018).

[2] D. Hanneke, S. Fogwell, and G. Gabrielse, New Measurement of the Electron Magnetic Moment and the Fine Structure Constant, Phys. Rev. Lett. 100, 120801 (2008).
[3] R. H. Parker, C. Yu, W. Zhong, B. Estey, and H. Müller, Measurement of the fine-structure constant as a test of the standard model, Science 360, 191 (2018).

[4] L. Morel, Z. Yao, P. Cladé, and S. Guellati-Khélifa, Determination of the fine-structure constant with 81 parts-per-trillion accuracy, Nature (London) 588, 61 (2020). 
[5] A. Matveev, C. G. Parthey, K. Predehl, J. Alnis, A. Beyer, R. Holzwarth, T. Udem, T. Wilken, N. Kolachevsky, M. Abgrall, D. Rovera, C. Salomon, P. Laurent, G. Grosche, O. Terra, T. Legero, H. Schnatz, S. Weyers, B. Altschul, and T. W. Hänsch, Precision Measurement of the Hydrogen $1 S-2 S$ Frequency via a 920-km Fiber Link, Phys. Rev. Lett. 110, 230801 (2013).

[6] V. A. Yerokhin, K. Pachucki, and V. Patkos, Theory of the Lamb shift in hydrogen and light hydrogen-like ions, Ann. Phys. (Berlin) 531, 1800324 (2019).

[7] E. J. Salumbides, J. C. J. Koelemeij, J. Komasa, K. Pachucki, K. S. E. Eikema, and W. Ubachs, Bounds on fifth forces from precision measurements on molecules, Phys. Rev. D 87, 112008 (2013).

[8] S. Alighanbari, G. S. Giri, F. L. Constantin, V. I. Korobov, and S. Schiller, Precise test of quantum electrodynamics and determination of fundamental constants with $\mathrm{HD}^{+}$ions, Nature (London) 581, 152 (2020).

[9] S. Patra, M. Germann, J.-Ph. Karr, M. Haidar, L. Hilico, V. I. Korobov, F. M. J. Cozijn, K. S. E. Eikema, W. Ubachs, and J. C. J. Koelemeij, Proton-electron mass ratio from laser spectroscopy of $\mathrm{HD}^{+}$at the part-per-trillion level, Science 369, 1238 (2020).

[10] S. Sturm, F. Köhler, J. Zatorski, A. Wagner, Z. Harman, G. Werth, W. Quint, C. H. Keitel, and K. Blaum, High-precision measurement of the atomic mass of the electron, Nature (London) 506, 467 (2014).

[11] F. Heiße, F. Köhler-Langes, S. Rau, J. Hou, S. Junck, A. Kracke, A. Mooser, W. Quint, S. Ulmer, G. Werth, K. Blaum, and S. Sturm, High-Precision Measurement of the Proton's Atomic Mass, Phys. Rev. Lett. 119, 033001 (2017).

[12] F. Heiße, S. Rau, F. Köhler-Langes, W. Quint, G. Werth, S. Sturm, and K. Blaum, High-precision mass spectrometer for light ions, Phys. Rev. A 100, 022518 (2019).

[13] D. J. Fink and E. G. Myers, Deuteron-to-Proton Mass Ratio from the Cyclotron Frequency Ratio of $\mathrm{H}_{2}^{+}$to $\mathrm{D}^{+}$with $\mathrm{H}_{2}^{+}$ in a Resolved Vibrational State, Phys. Rev. Lett. 124, 013001 (2020).

[14] S. Rau, F. Heiße, S. Köhler-Langes, F. Sasidharan, R. Haas, D. Renisch, C. E. Düllmann, W. Quint, S. Sturm, and K. Blaum, Penning trap mass measurements of the deuteron and the $\mathrm{HD}^{+}$ molecular ion, Nature (London) 585, 43 (2020).

[15] M. Hori, A. Sótér, D. Barna, A. Dax, R. Hayano, S. Friedreich, B. Juhász, T. Pask, E. Widmann, D. Horváth, L. Venturelli, and N. Zurlo, Two-photon laser spectroscopy of antiprotonic helium and the antiproton-to-electron mass ratio, Nature (London) $\mathbf{4 7 5}$, 484 (2011)

[16] M. Hori, H. Aghai-Khozani, A. Sótér, D. Barna, A. Dax, R. Hayano, T. Kobayashi, Y. Murakami, K. Todoroki, H. Yamada, D. Horváth, and L. Venturelli, Buffer-gas cooling of antiprotonic helium to 1.5 to $1.7 \mathrm{~K}$, and antiproton-to-electron mass ratio, Science 354, 610 (2016).

[17] U. Bressel, A. Borodin, J. Shen, M. Hansen, I. Ernsting, and S. Schiller, Manipulation of Individual Hyperfine States in Cold Trapped Molecular Ions and Application to $\mathrm{HD}^{+}$Frequency Metrology, Phys. Rev. Lett. 108, 183003 (2012).

[18] J. C. J. Koelemeij, B. Roth, A. Wicht, I. Ernsting, and S. Schiller, Vibrational Spectroscopy of $\mathrm{HD}^{+}$with 2-ppb Accuracy, Phys. Rev. Lett. 98, 173002 (2007).

[19] J. Biesheuvel, J.-Ph. Karr, L. Hilico, K. S. E. Eikema, W. Ubachs, and J. C. J. Koelemeij, Probing QED and fundamental constants through laser spectroscopy of vibrational transitions in $\mathrm{HD}^{+}$, Nat. Commun. 7, 10385 (2016).

[20] J. Biesheuvel, J.-Ph. Karr, L. Hilico, K. S. E. Eikema, W. Ubachs, and J. C. J. Koelemeij, High-precision spectroscopy of the $\mathrm{HD}^{+}$molecule at the 1-p.p.b. level, Appl. Phys. B Lasers Opt. 123, 23 (2017).

[21] S. Alighanbari, M. G. Hansen, V. I. Korobov, and S. Schiller, Rotational spectroscopy of cold and trapped molecular ions in the Lamb-Dicke regime, Nat. Phys. 14, 555 (2018).

[22] V. I. Korobov, L. Hilico, and J.-Ph. Karr, Fundamental Transitions and Ionization Energies of the Hydrogen Molecular Ions with Few ppt Uncertainty, Phys. Rev. Lett. 118, 233001 (2017).

[23] D. T. Aznabayev, A. K. Bekbaev, and V. I. Korobov, Leadingorder relativistic corrections to the rovibrational spectrum of $\mathrm{H}_{2}^{+}$ and $\mathrm{HD}^{+}$molecular ions, Phys. Rev. A 99, 012501 (2019).

[24] I. V. Kortunov, S. Alighanbari, M. G. Hansen, G. S. Giri, V. I. Korobov, and S. Schiller, Proton-electron mass ratio by high-resolution optical spectroscopy of ion ensembles in the resolved-carrier regime, Nat. Phys. 17, 569 (2021).

[25] E. Tiesinga, P. J. Mohr, D. B. Newell, and B. N. Taylor, The 2018 CODATA recommended values of the fundamental physical constants (Web version 8.1), http://physics.nist.gov/ constants.

[26] P. J. Mohr, D. B. Newell, and B. N. Taylor, CODATA recommended values of the fundamental physical constants: 2014 J. Phys. Chem. Ref. Data 45, 043102 (2016).

[27] R. Szafron, E. Y. Korzinin, V. A. Shelyuto, V. G. Ivanov, and S. G. Karshenboim, Virtual Delbrück scattering and the Lamb shift in light hydrogenlike atoms, Phys. Rev. A 100, 032507 (2019).

[28] S. G. Karshenboim, A. Ozawa, V. A. Shelyuto, R. Szafron, and V. G. Ivanov, The Lamb shift of the $1 s$ state in hydrogen: Two-loop and three-loop contributions, Phys. Lett. B 795, 432 (2019).

[29] See Supplemental Material at http://link.aps.org/supplemental/ 10.1103/PhysRevResearch.3.L022028 for updated frequency values and details on the theoretical calculation, hypothesis test, and combined constraints on NP.

[30] V. I. Korobov, Leading-order relativistic and radiative corrections to the rovibrational spectrum of $\mathrm{H}_{2}{ }^{+}$and $\mathrm{HD}^{+}$molecular ions, Phys. Rev. A 74, 052506 (2006).

[31] V. I. Korobov, Relativistic corrections of $m \alpha^{6}$ order to the rovibrational spectrum of $\mathrm{H}_{2}^{+}$and $\mathrm{HD}^{+}$molecular ions, Phys. Rev. A 77, 022509 (2008).

[32] V. I. Korobov, Calculation of the nonrelativistic Bethe logarithm in the velocity gauge, Phys. Rev. A 85, 042514 (2012).

[33] V. I. Korobov, L. Hilico, and J.-P. Karr, Theoretical transition frequencies beyond $0.1 \mathrm{ppb}$ accuracy in $\mathrm{H}_{2}^{+}, \mathrm{HD}^{+}$, and antiprotonic helium, Phys. Rev. A 89, 032511 (2014).

[34] V. I. Korobov, Coulomb three-body bound-state problem: Variational calculations of nonrelativistic energies, Phys. Rev. A 61 , 064503 (2000).

[35] J.-Ph. Karr, M. Haidar, L. Hilico, and V. I. Korobov, Precision calculations for three-body molecular bound states, in Recent Progress in Few-Body Physics, edited by N. A. Orr, M. Ploszajczak, F. M. Marques, and J. Carbonell, Springer Proceedings in Physics, Vol. 238 (Springer, Cham, 2020), pp. 75-81. 
[36] V. I. Korobov, J.-P. Karr, M. Haidar, and Z.-X. Zhong, Hyperfine structure in the $\mathrm{H}_{2}^{+}$and $\mathrm{HD}^{+}$molecular ions at order $m \alpha^{6}$, Phys. Rev. A 102, 022804 (2020).

[37] J.-Ph. Karr, M. Haidar, L. Hilico, Z.-X. Zhong, and V. I. Korobov, Higher-order corrections to spin-spin scalar interactions in $\mathrm{HD}^{+}$and $\mathrm{H}_{2}^{+}$, Phys. Rev. A 102, 052827 (2020).

[38] M. Borkowski, A. A. Buchachenko, R. Ciuryło, P. S. Julienne, H. Yamada, Y. Kikuchi, Y. Takasu, and Y. Takahashi, Weakly bound molecules as sensors of new gravity like forces, Sci. Rep. 9, 14807 (2019).

[39] N. Hölsch, M. Beyer, E. J. Salumbides, K. S. E. Eikema, W. Ubachs, C. Jungen, and F. Merkt, Benchmarking Theory with an Improved Measurement of the Ionization and Dissociation Energies of $\mathrm{H}_{2}$, Phys. Rev. Lett. 122, 103002 (2019).

[40] C. Delaunay, R. Ozeri, G. Perez, and Y. Soreq, Probing atomic Higgs-like forces at the precision frontier, Phys. Rev. D 96, 093001 (2017).

[41] C. Delaunay, C. Frugiuele, E. Fuchs, and Y. Soreq, Probing new spin-independent interactions through precision spec- troscopy in atoms with few electrons, Phys. Rev. D 96, 115002 (2017).

[42] I. Counts, J. Hur, D. P. L. Aude Craik, H. Jeon, C. Leung, J. C. Berengut, A. Geddes, A. Kawasaki, W. Jhe, and V. Vuletić, Evidence for Nonlinear Isotope Shift in $\mathrm{Yb}^{+}$Search for New Boson, Phys. Rev. Lett. 125, 123002 (2020).

[43] C. Solaro, S. Meyer, K. Fisher, J. C. Berengut, E. Fuchs, and M. Drewsen, Improved Isotope-Shift-Based Bounds on Bosons beyond the Standard Model through Measurements of the ${ }^{2} \mathrm{D}_{3 / 2}{ }^{2} \mathrm{D}_{5 / 2}$ Interval in $\mathrm{Ca}^{+}$, Phys. Rev. Lett. 125, 123003 (2020).

[44] Y. Kamiya, K. Itagaki, M. Tani, G. N. Kim, and S. Komamiya, Constraints on New Gravitylike Forces in the Nanometer Range, Phys. Rev. Lett. 114, 161101 (2015).

[45] C. C. Haddock, N. Oi, K. Hirota, T. Ino, M. Kitaguchi, S. Matsumoto, K. Mishima, T. Shima, H. M. Shimizu, W. M. Snow, and T. Yoshioka, Search for deviations from the inverse square law of gravity at $\mathrm{nm}$ range using a pulsed neutron beam, Phys. Rev. D 97, 062002 (2018). 